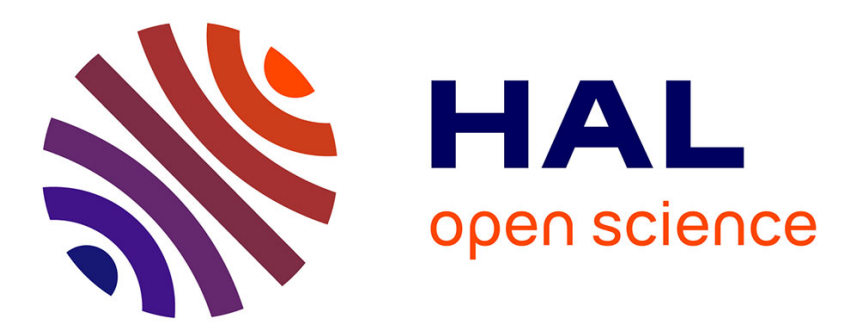

\title{
Optimal Sensor Configuring Techniques for The Compensation of Thermo-Elastic Deformations in High-Precision Systems
}

\author{
A.H. Koevoets, H.J. Eggink, J. van Der Sanden, J. Dekkers, T.A.M. Ruijl
}

\section{- To cite this version:}

A.H. Koevoets, H.J. Eggink, J. van Der Sanden, J. Dekkers, T.A.M. Ruijl. Optimal Sensor Configuring Techniques for The Compensation of Thermo-Elastic Deformations in High-Precision Systems. THERMINIC 2007, Sep 2007, Budapest, Hungary. pp.208-213. hal-00202562

HAL Id: hal-00202562

https://hal.science/hal-00202562

Submitted on 7 Jan 2008

HAL is a multi-disciplinary open access archive for the deposit and dissemination of scientific research documents, whether they are published or not. The documents may come from teaching and research institutions in France or abroad, or from public or private research centers.
L'archive ouverte pluridisciplinaire HAL, est destinée au dépôt et à la diffusion de documents scientifiques de niveau recherche, publiés ou non, émanant des établissements d'enseignement et de recherche français ou étrangers, des laboratoires publics ou privés. 


\title{
Optimal Sensor Configuring Techniques for the Compensation of Thermo-Elastic Deformations in High-Precision Systems
}

\author{
A.H. Koevoets, H.J. Eggink, J. van der Sanden, J. Dekkers and T.A.M. Ruij1 \\ e-mail corresponding author: A.H.Koevoets@philips.com \\ Philips Applied Technologies, High Tech Campus 7 \\ $5656 \mathrm{AE}$ Eindhoven, The Netherlands
}

\begin{abstract}
The time dependent thermal behaviour of precision systems and processes in relation to positioning accuracy is an important aspect and often claims a large part of the available position budged. This problem can be dealt with using real-time error-compensation method that combines measured temperatures with knowledge of thermo-elastic properties of the precision system. The thermally induced time varying deformations are calculated real-time by the error compensation model based on measured temperatures. The gained performance depends on the uncertainty of the thermomechanical model, the configuration of the temperature sensors and uncertainties in the temperature measurements. In the paper a method is introduced that enables optimization of the sensor configuration and accompanying compensation model based on the modal reduction technique. This method can be used in cases where only little pre-knowledge of heat-loads are available and enables a more optimal sensor placement, thus improving the performance of the error-compensation method.
\end{abstract}

\section{INTRODUCTION}

Accurate positioning is an important aspect of precision systems and processes, but the position performance is strongly influenced by transient thermal behaviour that results in deformations of the mechanical structure. There are various ways to deal with this unwanted phenomenon. Amongst these are using very low thermal expansion materials for the structure or thermal shielding/stabilisation by means of coolant flows. An alternative way is using real time temperature information of the structure to predict the thermo-mechanical deformations. These deformations can be seen as a kind of offset, which can be compensated for in the measuring and control software. This method is more generally known as error-compensation.

To make this method work several ingredients are necessary. First of all the relation between temperature distribution and deformation i.e. the thermo-mechanical coupling is required and can be a full FEM or reduced model. Secondly a number of temperature sensing points are required. The number of points must be as small as possible because of wiring, but large enough to give the required input for the error compensation model. This paper describes methods to obtain a more optimal sensor configuration and accompanying error compensation model that predicts the thermal induced deformations, therefore these unwanted deformations can be compensated.

To investigate these methods, an experimental test rig was build as shown in Fig. 1 and 2. This Aluminium frame has the outline dimensions $500 \times 355 \times 40 \mathrm{~mm}$. The gap distance has to be compensated by error-compensation. An (arbitrary) uniform heat load at the bottom is used to evaluate the performance of the error correction method.

This numerical case of Fig. 3 is a simplification of the test rig build to verify error compensation methods, see [1]. and Fig. 1 and 2. It consist of several types of material (with different coefficient of thermal expansion), intermediate bodies and it is equipped with 52 temperature sensors. To measure the internal deformation the frame is also equipped with 3 capacitive gauges, this displacement measurement is used to validate the error-compensation method.

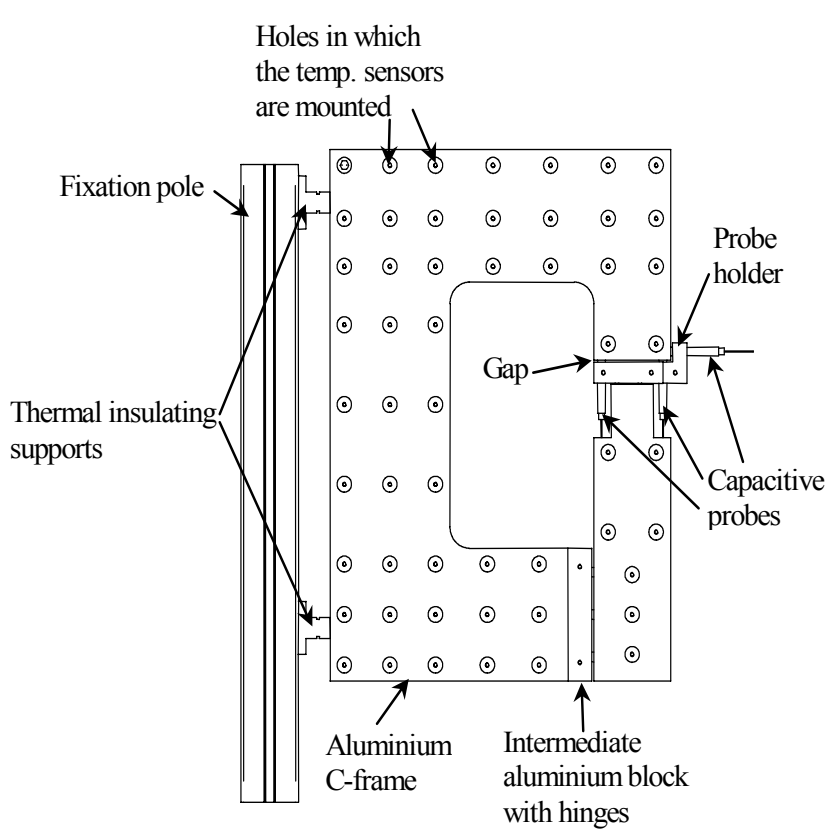

Fig. 1 - Schematic set-up of C-frame 


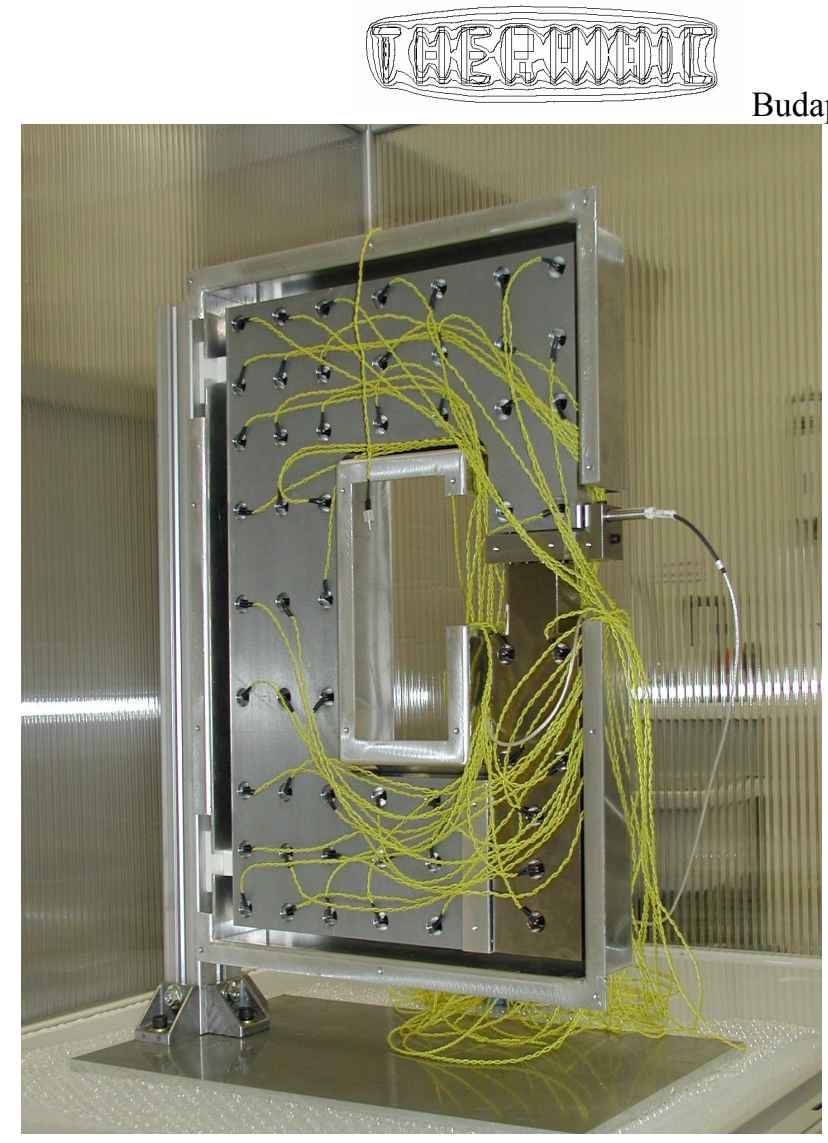

Fig. 2 - C-frame set-up with sensors

Heat loads working on the $\mathrm{C}$-frame will induce time varying temperature distributions causing time varying deformations. For devices used in high accuracy processes we are mainly interested in deformations of the device that is important for our process, in this paper the gap distance, depicted as performance measure in Fig. 3. A number of temperature sensors should be placed at certain positions on the C-frame such that they are able to (approximately) reconstruct the important part of the time varying temperature distribution, needed to predict the relevant deformation.

The problem that is dealt with in this paper is how to obtain the most accurate error compensation model, and derive guidelines for the number of sensors to be used and optimize their position on the frame. In this paper an accurate error compensation model and design rules for sensor configuration is derived using the modal reduction method. Although not shown in this paper good results are also expected using Arnoldi-like or Proper Orthogonal Decomposition (POD) methods, see [2]. , [3]. and [4]. for these methods. But in this work we assume to have only little pre-knowledge of the disturbing heat-load and this limitation rules these two methods out.

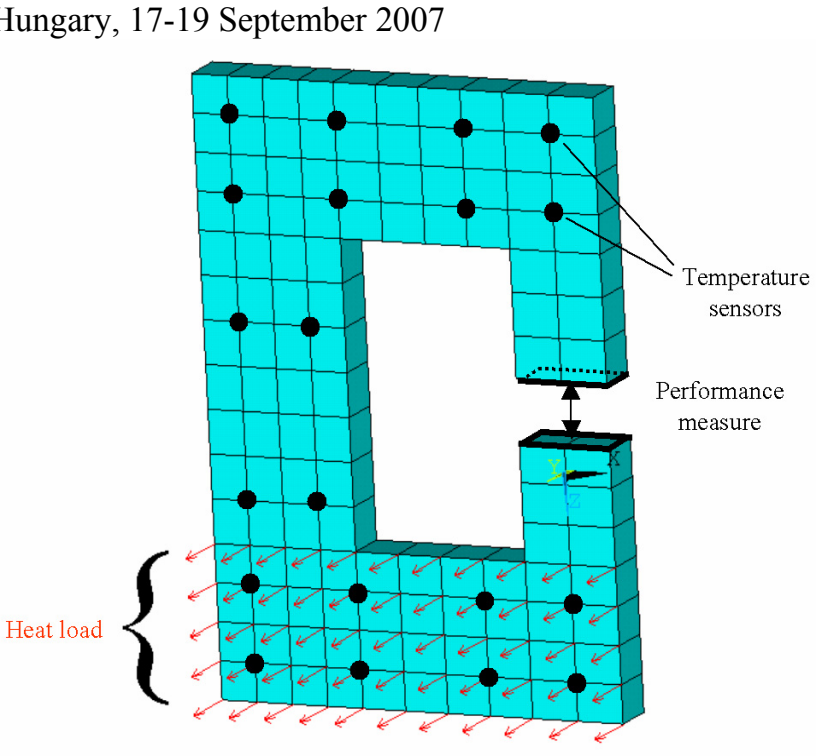

Fig. 3 FEM-model of an Al C-frame a uniform load and the position of temperature sensors are shown

In this paper we describe a method that uses mainly the properties of the $\mathrm{C}$-frame in a dynamic sense (i.e. its thermal capacity and conduction properties). This method uses a modal analysis of the thermo-elastic system properties and is therefore called 'modal-compensation'. Both compensation methods are evaluated using the simplified numerical model of the C-frame, see Fig. 3.
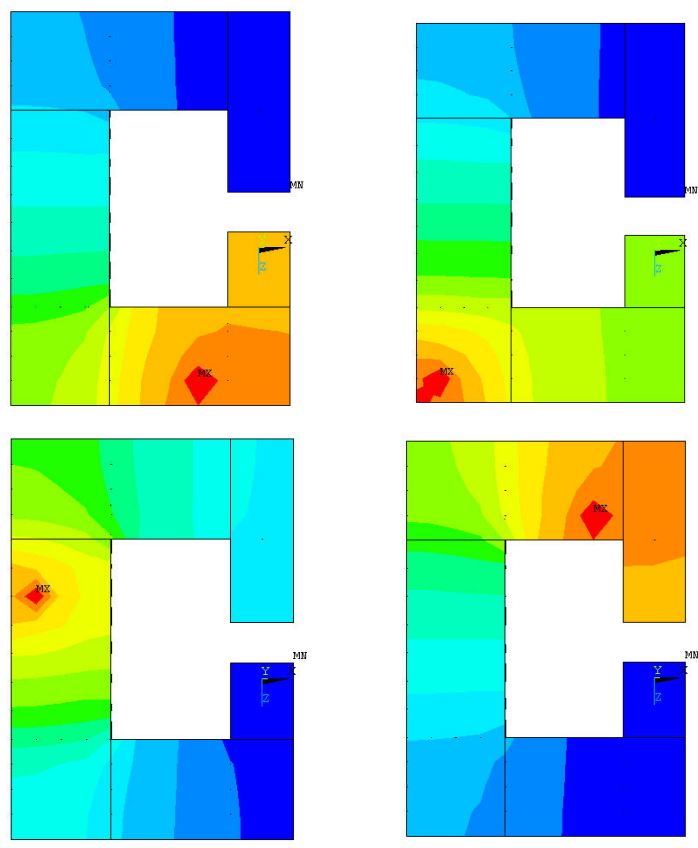

Fig. 4. static thermal response of point loads on different sensor locations. 


\section{NODAL COMPENSATION METHOD}

If we neglect inertia effects, the relation between temperature and deformation is static, therefore the frame deformations can be calculated at every point in time using a linear weighted summation of all temperature DOF (Degrees Of Freedom) at that same point in time

$$
p(t)=\left[r_{1} \cdots r_{n}\right]\left[\begin{array}{c}
T_{1}(t) \\
\vdots \\
T_{n}(t)
\end{array}\right]
$$

Here $p(t)$ is the relevant deformation i.e. gap displacement as a function of time, $\left[r_{1} \ldots r_{n}\right]$ is the sensitivity vector containing the thermo-elastic coupling and $T_{i}$ the temperature measured using sensor $i$. Now $n$ is the number of DOF of our FEM representation of the $\mathrm{C}$-frame and a large number of 400 is used in our numerical case, in fact this is a approximation for the infinite number of the continuous system. Using $n$ temperature sensors (a very large number of $n=400$ ) is both impractical and expensive, a small set of $m<<n$ sensors (20 in our case) is distributed over the C-frame (see Fig. 3). Now we need to find the $m$ coefficients of $\left[r_{1} \cdots r_{m}\right]$, determining the relation between the $m$ temperature sensors and the gap distance $p$. The $m$ unknowns require $m$ equations, meaning $m$ independent temperature fields with their corresponding gap distance $p$. So we get in

$$
\left[\begin{array}{lll}
p_{1} & \cdots & p_{m}
\end{array}\right]=\left[r_{1} \cdots r_{m}\right]\left[\begin{array}{ccc}
T_{1,1} & \cdots & T_{1, m} \\
\vdots & & \vdots \\
T_{m, 1} & \cdots & T_{m, m}
\end{array}\right]
$$

or in matrix notation $\mathbf{p}=\mathbf{r} \mathbf{T}$.

The temperature fields are generated using $m$ different point loads on the $m$ sensor positions of the C-frame, see Fig. 4. The coefficients of $\mathbf{r}$ (the error-compensation model) can be calculated as

$$
\mathbf{r}=\mathbf{p} \mathbf{T}^{-1}
$$

This method is evaluated by comparing the "true" transfer -function of heat load to gap displacement (based on 400 DOF FEM-model) with the error-compensation model (20 DOF, nodal method). The transfer functions are shown in Fig. 5. This nodal-compensation method performs well at low frequencies (static) but for higher frequencies the accuracy of the prediction decreases. The reason of this uncertainty is the fact that the 400 DOF of the entire FEM model are approximated using only 20 DOF. If other temperature distributions are used to calculate $\mathbf{r}$, a different model is obtained and a different estimation of the behaviour. The better the distributions are able to represent the true (temperature) distributions as they occur the better the error-compensation will perform. Note that this is the reason that a
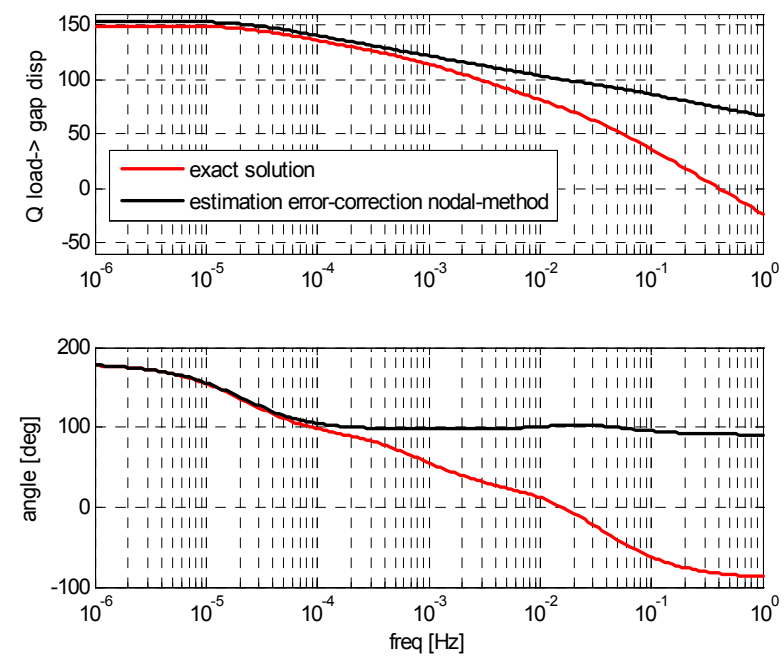

Fig. 5. FRF load Q-> gap distance p, FEM-solution vs. nodal errorcompensation

POD based method will most likely outperform other methods in the cases where the actual heat-load and accompanying response resemble the ones used during the identification. Because the exact features of our heat load are assumed to be unknown we focus on known system properties like capacity, conduction and thermo-mechanical coupling properties. It is expected that the thermo-elastic behaviour is dominated by only a few modes, therefore in chapter 4 the thermal mode-shapes are used to find a compensation model that focuses on reconstructing the total temperature distribution using the mode-shapes that are identified or (when we have less pre-knowledge) expected to be important.

\section{MODAL COMPENSATION METHOD}

The transient thermal-elastic behaviour of the $\mathrm{C}$-frame with a heat-load shape $\mathbf{Q}_{(\mathrm{n} \times 1)}$, heat load amplitude $u(t)_{\left(\begin{array}{ll}1 & \times\end{array}\right)}$ can be written using first order differential equation

$$
\mathbf{C} \dot{\mathbf{T}}(t)+\mathbf{K T}(t)=\mathbf{Q} u(t) \text { or } \dot{\mathbf{T}}(t)=-\mathbf{C}^{-1} \mathbf{K T}(t)+\mathbf{C}^{-1} \mathbf{Q} u(t)
$$

And the corresponding deformation gap distance $p(t)_{(1 \times 1)}$ by

$$
p(t)=\mathbf{S}_{\mathbf{P}-\mathbf{T}} \mathbf{T}(t)
$$

With $\mathbf{C}_{(\mathrm{n} \times \mathrm{n})}$ the capacity matrix, $\mathbf{K}_{(\mathrm{nxn})}$ the conduction matrix, $\mathbf{T}_{(\mathrm{n} \times 1)}$ the vector containing the $\mathrm{n}$ time varying temperature DOF (and not equal to $\mathbf{T}$ as used in (2) and (3) and $\left.\mathbf{S}_{\mathbf{P}-\mathbf{T}(1 \times} \times \mathrm{n}\right)$ the thermo-mechanical coupling matrix. Without loss of generality we can rewrite (4) and (5) into the modal form of (6) using the modal transformation $\mathbf{T}(t)=\boldsymbol{\Phi q}(t)$, where $\boldsymbol{\Phi}_{(\mathrm{n} \times \mathrm{n})}$ contains the eigenvectors (i.e. mode-shapes) of the system matrix $-\mathbf{C}^{-1} \mathbf{K}$. And $\mathbf{q}(\mathrm{t})$ (n $\left._{\mathrm{x}} \quad 1\right)$ contains the modal responses. 


$$
\begin{aligned}
\dot{\mathbf{q}}(t) & =\boldsymbol{\Phi}^{-1}\left[-\mathbf{C}^{-1} \mathbf{K}\right] \mathbf{\Phi} \mathbf{q}(t)+\boldsymbol{\Phi}^{-1} \mathbf{C}^{-1} \mathbf{Q} u(t) \\
p(t) & =\mathbf{S}_{\mathbf{P}-\mathbf{T}} \mathbf{\Phi} \mathbf{q}(t)
\end{aligned}
$$

The first 3 thermo-mechanic mode-shapes are plotted in Fig. $6 \mathrm{a}, 6 \mathrm{~b}$ and $6 \mathrm{c}$ (the modes are ranked by decreasing timeconstant), where the temperature values are represented by an out of plane displacement. In (6), $\boldsymbol{\Phi}^{-1}\left[-\mathbf{C}^{-1} \mathbf{K}\right] \boldsymbol{\Phi}$ is the modal system matrix a diagonal matrix containing the inverses of the $n$ time-constants $\left(\tau_{i}\right)$. Because capacity $\mathbf{C}$ has only non-zero values on its diagonal and $\mathbf{K}$ is symmetric $-\mathbf{C}^{-1} \mathbf{K}$ will have real eigenvalues. Because $\boldsymbol{\Phi}^{-1}\left[-\mathbf{C}^{-1} \mathbf{K}\right] \boldsymbol{\Phi}$ is a diagonal matrix (6) can be rewritten into $n$ independent differential equations. [ $\left.\boldsymbol{\Phi}^{-1} \mathbf{C}^{-1} \mathbf{Q}\right]$ is the so-called modal controllability. The amplification of the heat load on the different modes is shown by $\left(\left[\begin{array}{lll}\phi_{\text {modal cont } 1} & \cdots & \phi_{\text {modal cont } \mathbf{n}}\end{array}\right]^{T}\right)$, in Fig.. 7a we can see that especially the first modes are sensitive for the load $u(t)$. The row $\left[\mathbf{S}_{\mathbf{P - T}} \boldsymbol{\Phi}\right]$ is the so-called modal observability $\left[\begin{array}{lll}\phi_{\text {modal observ } 1} & \cdots & \phi_{\text {modal observ n }}\end{array}\right]$ that shows the influence of the different modes on the gap distance, this modal observability is plotted in Fig.. 6b. Now using the Laplace operator $s$, (6) can be rewritten as the summation of $n$ independent modal contributions

$$
p(s)=\sum_{i=1}^{n} \frac{\phi_{\text {modal observ i }} \cdot \phi_{\text {modal cont i }}}{s-\frac{1}{\tau_{i}}} \cdot u(s)
$$

In the previous chapter the question raised how to reconstruct an $n$ dimensional temperature field using only $m$ sensors when $m<<n$ (in our case $20<<400$ ). In Fig. 7a we see that the temperature field is mainly determined by some of the first 10 modes. This will depend on the spatial smoothness of the heat-load, e.g. when shielding is used we can expect it to be more likely that only the first modes with large spatial features are dominant over those with small spatial features. In Fig.. $7 \mathrm{~b}$ we see that only some of the first 40 modes have a dominant effect on the gap distance. In Fig. $7 \mathrm{c}$ the product of Fig. $7 \mathrm{a}$ and $7 \mathrm{~b}$ is plotted and this shows which modes are both significantly excited and important for the gap distance. This knowledge can be used to determine the number of sensors needed for error-compensation, i.e. the number of sensors is at least equal to the number of important modes. The (initial) spatial distribution of the sensors over the $\mathrm{C}$-frame can be derived looking at the shape of these important modes. Note that for a frame with a homogenous thickness made of only one material an equidistant grid like shown in Fig. 1 is a sensible (initial) sensor configuration.

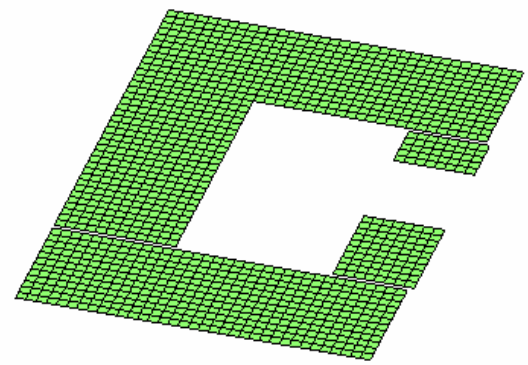

Fig. 6a. Mode-shape 1 Magnetization as a

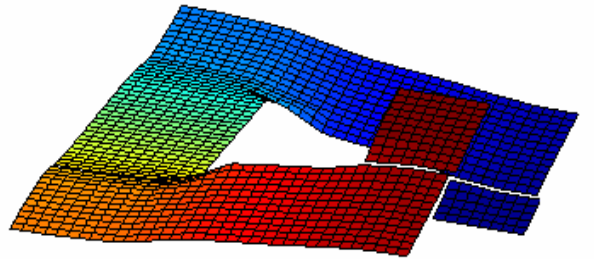

Fig. 6b. Mode-shape 2

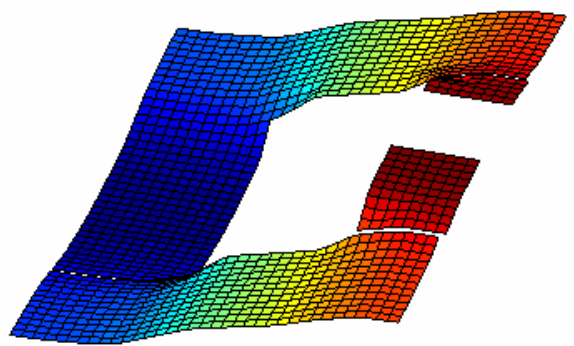

Fig. 6c. Mode-shape 3

From (7) and Fig. 6c we know that only a few modes dominate the relation between heat load and gap distance, therefore we choose to use a sensor error-compensation model combination that is able to approximately reconstruct the total $n$ dimensional temperature distribution using these dominant mode-shapes. Now the $n$ nodal displacements can be estimated using $m$ sensors, with $m<<n$ using (8)

$$
\left[\begin{array}{c}
\hat{q}_{1}(t) \\
\vdots \\
\hat{q}_{n}(t)
\end{array}\right]=\left[\begin{array}{ccc}
\alpha_{1,1} & \cdots & \alpha_{1, m} \\
\vdots & & \vdots \\
\alpha_{n, 1} & \cdots & \alpha_{n, m}
\end{array}\right]\left[\begin{array}{c}
T_{1}\left(x_{1}, y_{1}, t\right) \\
\vdots \\
T_{m}\left(x_{m}, y_{m}, t\right)
\end{array}\right]
$$

The temperature vector $\mathbf{T}$ containing the true measured temperatures can be written (without loss of generality) as

$$
\left[\begin{array}{c}
T_{1}\left(x_{1}, y_{1}, t\right) \\
\vdots \\
T_{m}\left(x_{m}, y_{m}, t\right)
\end{array}\right]=\left[\begin{array}{ccc}
\phi_{1,1}\left(x_{1}, y_{1}\right) & \cdots & \phi_{1, n}\left(x_{1}, y_{1}\right) \\
\vdots & & \vdots \\
\phi_{m, 1}\left(x_{m}, y_{m}\right) & \cdots & \phi_{m, n}\left(x_{m}, y_{m}\right)
\end{array}\right] \cdot\left[\begin{array}{c}
q_{1}(t) \\
\vdots \\
q_{n}(t)
\end{array}\right]
$$

Combining (8) and (9) results in 


$$
\hat{\mathbf{q}}(t)=\mathbf{A} \boldsymbol{\Phi}(\mathbf{x}, \mathbf{y}) \mathbf{q}(t)
$$

Here $\hat{q}_{i}$ is the estimation of the modal displacement $q_{i}$ of mode $\mathrm{i}, \alpha_{i, j}$ is the coefficient for weighing the temperature signal $T_{j}\left(x_{j}, y_{j}, t\right)$ measured by sensor $j$ to obtain the estimation of modal displacement $i$. These temperatures $\mathbf{T}$ are a function of both time and position and can be rewritten using $\mathbf{T}(\mathbf{x}, \mathbf{y}, t)=\mathbf{\Phi}(\mathbf{x}, \mathbf{y}) \mathbf{q}(t)$. Now $\phi_{j, i}\left(x_{j}, y_{j}\right)$ is the modal leverage of mode $i$ as observed at position $\left(x_{j}, y_{j}\right)$ by sensor $j$.

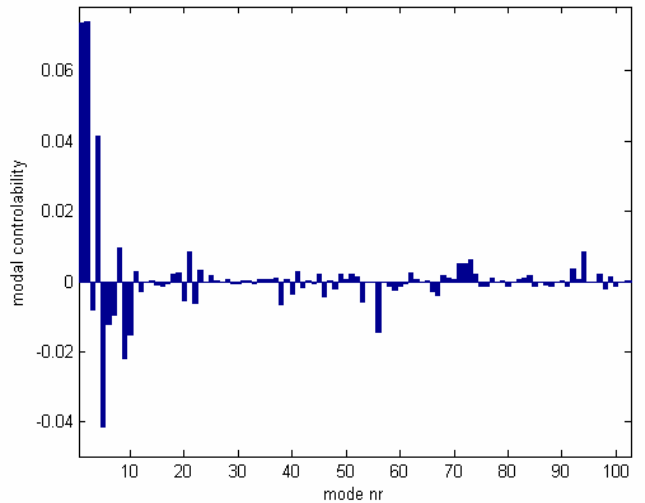

Fig. 7a. Modal controllability

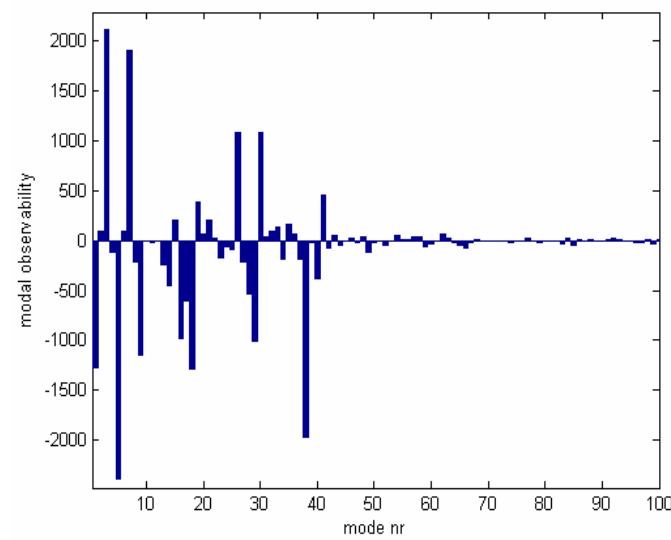

Fig. 7b. Modal observability

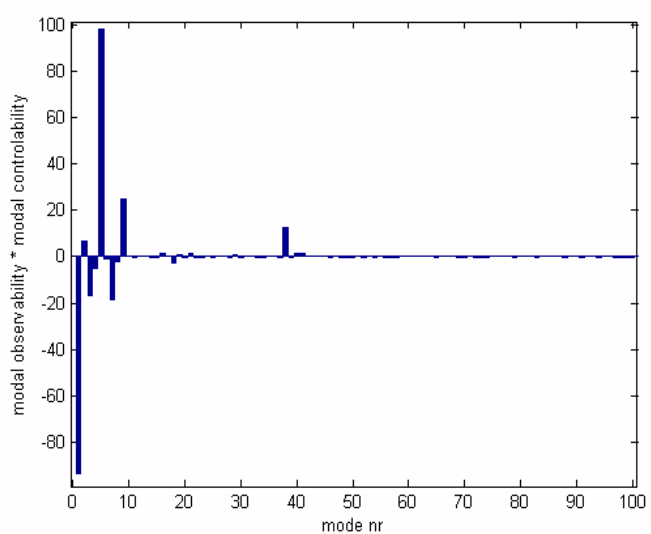

Fig. 7c. Modal controllability*observability
In (8) we can see that if $\mathbf{A}=\boldsymbol{\Phi}(\mathbf{x}, \mathbf{y})^{-1}$ the $n$ modal displacements are estimated exactly, but this inverse requires that $\boldsymbol{\Phi}(\mathbf{x}, \mathbf{y})$ is square, i.e. we need at least $n$ sensors.

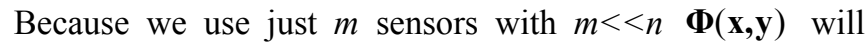
become rectangular and a so called pseudo inverse should be used, see (11)

$$
\mathbf{A}(\mathbf{x}, \mathbf{y})=\Phi(\mathbf{x}, \mathbf{y})^{\mathrm{T}}\left(\boldsymbol{\Phi}(\mathbf{x}, \mathbf{y}) \Phi(\mathbf{x}, \mathbf{y})^{\mathrm{T}}\right)^{-1}
$$

A contains the coefficients estimating the $\mathrm{n}$ different modal displacements. In (11) the effort of predicting the different $n$ modal displacements correctly is equally distributed over all the $n$ modes. Because we saw in Fig. 7 that only some modes are dominant for the systems behavior, hence more effort should be put in estimating the amplitudes of those modes that are important for the behavior, this can be enforced using a weighted pseudo inverse:

$$
A(x, y)=W \Phi(x, y)^{T}\left(\Phi(x, y) W \Phi(x, y)^{T}\right)^{-1}
$$

For our numerical case of Fig. 3 we apply a weighing factor of 1 for the first 20 modes and a 0 for the modes 21 up to 400. This resulted in improved error-compensation with respect to the so-called nodal-method, see Fig. 6.

A large improvement with respect to static estimation is obtained by using weighing factors such that the errorcompensation model focuses completely on estimating the first 20 modal displacements correctly (these are then multiplied with first 20 modal observability values of Fig. $7 \mathrm{~b}$ to obtain the gap distance). If these weighing factors are optimal depends on our level of pre-knowledge on the load, the sensitivity of the gap distance, the frequency content of the excitation signal and the time constraints of the application. For example if shielding is used the first modes with large spatial features will be dominate the behaviour and should be included. The effect of different modes on thermal induced deformation is often known in advance and can be modelled relatively accurate. We should include the modes that have the largest impact on the performance measure.

The coefficients of $\mathbf{A}(\mathbf{x}, \mathbf{y})$ as derived in (10) depend on the weighing factors $\mathbf{W}$ and the positions of the sensors $(\mathbf{x}, \mathbf{y})$. It is already mentioned that the sensor positions should be chosen such that the important modes are observed and distinguished, which could be done by looking at the important modes. When arbitrary sensor positioning is used, it can occur that different important modes are observed virtually the same. The corresponding columns in $\boldsymbol{\Phi}(\mathbf{x}, \mathbf{y})$ will become (virtually) similar and the coefficients of matrix $\mathbf{A}(\mathbf{x}, \mathbf{y})$ will have large differences i.e. the condition number of $\mathbf{A}(\mathbf{x}, \mathbf{y})$ becomes large. This means that some sensor signals are multiplied with huge coefficients making the 
error-compensation configuration more sensitive for measurement noise. This problem can be solved using numerical optimization that employs the sensor position DOF to minimize the condition number of matrix $\mathbf{A}(\mathbf{x}, \mathbf{y})$

$$
\min _{\mathbf{x}, \mathbf{y}} \kappa(\mathbf{A}(\mathbf{x}, \mathbf{y}))
$$

This is one example of using the sensor position DOF for improving the error-compensation's performance; other objectives are possible (e.g. insensitivity for certain modeshapes) and are the topic of future investigation.

The performance of error-compensation depends on the number of sensors, their positioning, how these signals are combined. But also practical limitations such as the accuracy of temperature sensors and measurement noise are possible limitations for the error correction performance. Currently the setup shown in Fig. 1 and 2 is subjected to a sensitivity analysis where we zoom in on effects like heat generated by temperature sensors and the measurement bias caused by the effect of the ambient temperature on temperature sensor signal.
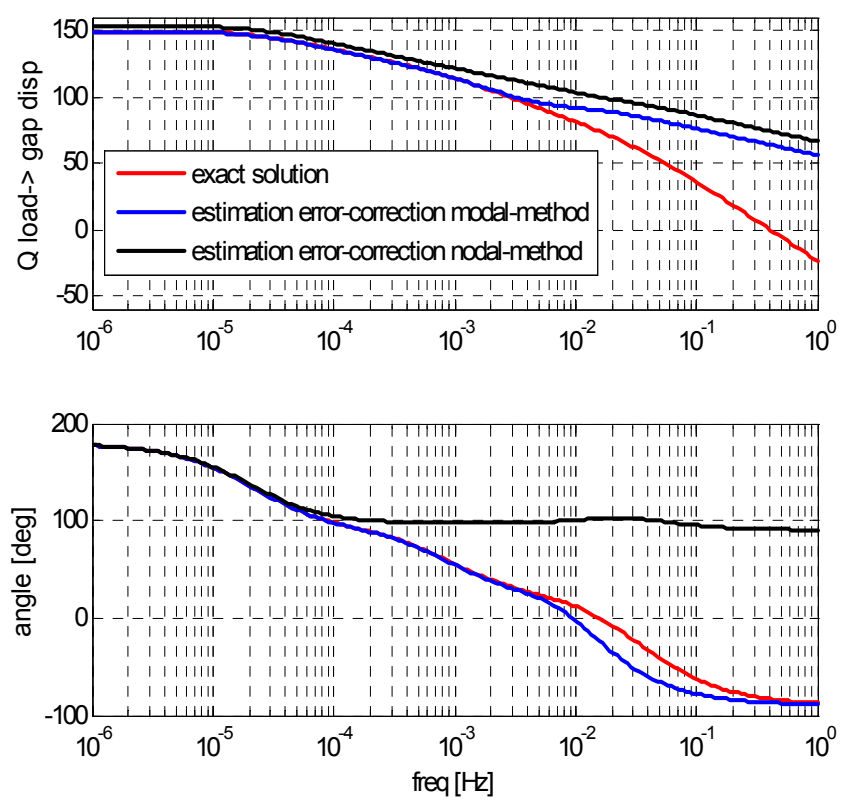

Fig. 8.FRF load Q-> gap distance p. FEM-solution vs. error compensation modal and nodal.

\section{CONCLUSIONS}

It is shown that modal analysis of the thermo-mechanical system properties can be used to derive the required number of sensors and an indication for preferred sensor positions and how to combine the sensor signals in an errorcompensation model. Although the practical merits of this technique should be explored using the experimental setup of Fig. 1 and 2, the numerical results already show large improvements with respect to known methods.
The advantage of this modal-based error-correction and sensor configuration optimization is that system knowledge i.e. capacity and conduction properties is used in combination with limited pre-knowledge of heat-loads to obtain well argued design criteria for the sensor configuration and corresponding model. The validation and implementation of this modal-based method will be tested and validated on the experimental test-rig.

Optimization criteria for the sensor DOF are proposed to enforce an error-compensation that is less sensitive for measurement noise. Possibilities of this optimization technique will be subject of future investigations at Philips Applied Technologies.

\section{References}

1. T.A.M. Ruijl, 'Ultra Precision Coordinate Measuring Machine', Ph.D. Thesis, TU-Delft, 2001.

2. R.W. Freund, Reduced-Order Modeling Techniques Based on Krylov subspaces and Their Use in Circuit Simulation, Numerical analysis Manuscript, 98-3-02, 1998

3. I.M. Elfadel and David D. Ling, A Block Rational Arnoldi Algorithm for Passive Model-Order Reduction of Multiport RLC Networks. Proc. ICCAD, 1997.

4. S.Vokwein. Proper Orthogonal Decomposition and Singular Value Decomposition. SFB-Preprint No 153, 1999. 